\title{
Comprehensive treatments for social cognitive deficits in schizophrenia: A critical review and effect-size analysis of controlled studies
}

Matthew M. Kurtz; Emily Gagen; Nuno B.F. Rocha; Sergio Machado; David L. Penn

\begin{abstract}
Recent advances in psychosocial treatments for schizophrenia have targeted social cognitive deficits. A critical literature review and effect-size (ES) analysis was conducted to investigate the efficacy of comprehensive programs of social cognitive training in schizophrenia. Results revealed 16 controlled studies consisting of seven models of comprehensive treatment with only three of these treatment models investigated in more than one study. The effects of social cognitive training were reported in $11 / 15$ studies that included facial affect recognition skills ( $E S=.84$ ) and $10 / 13$ studies that included theory-of-mind $(E S=.70)$ as outcomes. Less than half $(4 / 9)$ of studies that measured attributional style as an outcome reported effects of treatment, but effect sizes across studies were significant $(E S s=.30-.52)$. The effect sizes for symptoms were modest, but, with the exception of positive symptoms, significant (ESs $=.32-.40$ ). The majority of trials were randomized (13/16), selected active control conditions $(11 / 16)$ and included at least 30 participants (12/16). Concerns for this area of research include the absence of blinded outcome raters in more than $50 \%$ of trials and low rates of utilization of procedures for maintaining treatment fidelity. These findings provide preliminary support for the broader use of comprehensive social cognitive training procedures as a psychosocial intervention for schizophrenia.
\end{abstract}

\section{Keywords}

Social cognition; Psychosocial treatments; Affect recognition; Theory-of-mind; Schizophrenia

Functional impairment is one of the hallmarks of schizophrenia, required for DSM5 diagnosis and has implications for an individual's likelihood of relapse, course of illness, and overall quality of life, both subjective and objective (e.g., Edmondson et al., 2012 and Robertson et al., 2014). Social cognition has been identified as one of the major disorder features that underlie these impairments (Couture, Penn, \& Roberts, 2006), and includes the ability of individuals to understand themselves and others in the wider context of social interactions, especially others' thoughts, feelings, and intentions (Adolphs, 
$\underline{2009}$ and Fiske, 1991). NIMH's consensus statement, generated by a convention of leading social cognitive researchers, recognized theory of mind, emotion perception/processing, attributional style, social perception, and social knowledge as representing the major domains of social cognition (Green et al., 2008). Penn, Sanna, and Roberts (2008) similarly identified theory of mind, emotion perception, and attributional style as being particularly salient for individuals with schizophrenia, who consistently demonstrate impaired social cognitive abilities in each of these areas (e.g.,Mancuso et al., 2011, Pinkham et al., in press and Savla et al., 2013).

These deficits have engendered increased experimental investigation over the past 15 years for several reasons. First, research has indicated that these deficits are separable from those of neurocognition (Nuechterlein et al., 2004). Second, these deficits have strong and independent relationships to functional outcomes (Fett, Viechtbauer, Penn, van Os, \& Krabbendam, 2011). Third, emerging research suggests that these deficits may be more proximal to some dimensions of functional outcomes than deficits in neurocognition. For example, in a literature review and presentation of their own data, Schmidt, Mueller, and Roder (2011) demonstrated that social cognition served in many cases as a robust mediator of the relationship between neurocognition and functional outcome. Thus, social cognition has been identified as a vitally important area of research in schizophrenia; it is a means of exploring both the interpersonal difficulties that individuals with this illness experience, as well as the consequences of these difficulties, such as poorer vocational outcomes, a lack of community participation and independence, and limitations in the formation and maintenance of close emotional relationships (Couture et al., 2006). In total, these findings bolster rationales for devising treatments that target social cognitive deficits with a goal of generalized improvements in social functioning.

Many evidence-based psychosocial interventions for schizophrenia may influence social cognition, but do not typically directly target social cognition. For example, social skills training helps individuals to acquire and practice specific behavioral skills in social interactions, but does not require individuals to recognize, monitor, and practice skills in implementing underutilized social cognitive processes. CBT for psychosis (CBTp) targets the maladaptive thoughts and behaviors that individuals with schizophrenia often possess. Cognitive remediation aims to address impairment in information processing skills as a means of indirectly improving social functioning and other aspects of functional outcome. There has also been little support for the impact of existing pharmacological interventions for symptoms on social cognitive deficits (Harvey, Patterson, Potter, Zhong, \& Brecher, 2006). 
In recent years, there has been growth in the development and preliminary assessment of psychosocial treatment aimed directly at social cognitive deficits in schizophrenia. Initial "proof-of-concept" studies for the malleability of social cognitive processes have been positive. In addition to these targeted programs, a few treatment packages have included social cognitive training exercises as one element of much broader training programs that target a variety of dimensions of the illness; the two most well-represented examples of this type of approach in the research literature are Integrated Psychological Therapy (IPT; Brenner et al., 1994) and Cognitive Enhancement Therapy (CET; Hogarty et al., 2004). Though the efficacy of IPT for improving neurocognition, psychosocial functioning, and symptoms has been well-established (Roder, Mueller, Mueser, \& Brenner, 2006), and some recent studies have revealed effects of IPT on social cognitive outcomes (Roder, Mueller, \& Schmidt, 2011), the complexity of the intervention precludes linkage of specific training modules to specific outcomes. CET (Hogarty et al., 2004) is another multi-element treatment package that includes extensive social cognitive training along with cognitive remediation. Results from randomized controlled trials have shown that improvements in social cognition, as measured by clinician ratings on the Social Cognition Profile, were evident after 24 months of treatment, and a follow-up report indicated that these improvements persisted at 12 months after the cessation of treatment (Hogarty, Greenwald, \& Eack, 2006). It remains difficult to directly attribute this improvement to the social cognitive training, since it was just one component of a much larger treatment package targeting a number of disparate outcomes.

In recent years there has been substantial growth in the development, implementation and assessment of novel, integrated and comprehensive programs of social cognitive training. These programs extend beyond brief interventions for a single aspect of social cognition, devote all elements of an extended training program to enhancement of multiple domains of social cognition, and typically include practice for generalization of acquired skills to everyday life. Importantly, these programs provide information on the efficacy of social cognitive training for social cognitive processes and social functioning in the absence of the administration of additional, complementary evidence-based psychosocial interventions such as cognitive remediation or social skills training, which could be burdensome for resource-limited mental health clinics and for clients.

Several narrative reviews of social cognitive training in schizophrenia have been conducted (Choi et al., 2009, Fiszdon and Reddy, 2012, Horan et al., $\underline{2008}$ and Wolwer et al., 2010) and all have shown that social cognitive training 
produces effects on various aspects of social cognition, with more substantial effects on its more elementary aspects (i.e., affect perception and discrimination). In the most recent critical review of the literature, Fiszdon and Reddy (2012), on the basis of nearly 50 empirical studies, concluded that social cognitive training programs were most effective when focused on the extended practice of elementary social cognitive skills in which simple associations are formed between elements of facial expression (e.g. scrunched eyebrows) and an emotion (anger) and were considerably less effective when they placed high demands on elementary cognitive operations such as sustained attention and memory.

The only meta-analysis conducted in this area was based on a sample of 692 clients and revealed moderate to large effects of social cognitive training on facial affect recognition (identification $d=.71$, discrimination $d=1.01$ ) and theory-ofmind $(d=.46)$, and moderate to large effects on community and institutional functioning $(d=.78)$ and total symptoms $(d=.68)$ (Kurtz \& Richardson, 2012). However, this meta-analysis combined the results of brief proof-of-concept social cognitive interventions, interventions that include social cognition as one element of multi-element psychosocial treatments and comprehensive programs of social cognitive training. Thus, the efficacy of comprehensive social cognitive programs remains unknown. And, while Fiszdon and Reddy (2012) included an analysis of comprehensive social cognitive treatment programs in their analysis of "broadbased" programs of social cognition, they did not provide a quantitative analysis of these treatment effects via the analysis of study effect-sizes. In addition, a growing number of studies, the majority of high design quality (e.g.,Roberts et al., 2014), have been published over the past three years. Indeed, eight controlled trials of comprehensive programs of social cognitive training consisting of 378 participants have been published since the Kurtz \& Richardson, 2012 review, while 6 novel trials of social cognitive training consisting of 281 participants have been published since the Fiszdon \& Reddy, 2012 review. These new trials have represented several of the largest sample studies in this research area to date (e.g., Hasson-Ohayon et al., 2014 and Roberts et al., 2014).

The purpose of the current paper is to provide a critical analysis of the extant literature on comprehensive programs of social cognitive training. Specifically, this review will examine: 1) whether time and effort-intensive comprehensive social cognitive training programs improve social cognitive function; 2) whether these programs are effective for only specific domains of social cognitive function or whether they are able to influence multiple domains of social cognitive function simultaneously; and 3) whether any observed effects of social cognitive training extend more broadly to other measures of symptomatology. A secondary goal 
was to examine whether social cognitive training programs can improve neurocognition. On the one hand, it might be hypothesized that, for example, targeted practice on facial affect recognition tasks, and/or acquisition of skills in generating alternative hypotheses for social situations characteristic of many comprehensive social cognitive training programs might modify underlying neurocognitive skills, such as attention and cognitive flexibility, and that, in turn, these changes in elementary neurocognition might drive observed changes on measures of social cognition. Alternatively, it could be that these programs treat social cognitive deficits directly with little influence on underlying neurocognitive skills. Lastly, we provide an analysis of aggregate effect-sizes from the reviewed studies to provide a quantitative assessment of social cognitive training program effects.

We hypothesized that social cognitive training programs would produce effects on several domains of social cognition that they target including facial affect recognition, social perception, theory-of-mind and attributional style. We expected that these effects would generalize with small to moderate effects on symptoms.

\section{Methods}

\subsection{Search strategy}

Articles included in this analysis were identified through a computer-based search of Google Scholar using combinations of the following keywords: SCIT, SCST, "social cognition and interaction training", "social cognitive training", and schizophrenia. A parallel search using the same key terms was completed with the MEDLINE (National Library of Medicine, 1994) database from 1980 to 2014. Nineteen-eighty were selected as a cut-off in light of the introduction of the DSMIII for more reliable diagnostic criteria for schizophrenia (American Psychiatric Association, 1987). The reference sections of articles located from both searches were studied for relevant citations.

Articles were included if they met the following criteria: (a) studied a comprehensive program of social cognitive training focused on a minimum of two domains of social cognition, (b) did not include other psychosocial treatments as part of the intervention (e.g., cognitive remediation, social skills training), (c) included at least one standardized measure of social cognition as an outcome measure, (d) included a control group, (e) was published between 1980 and 2014, (f) was published in a peer-reviewed English language journal, and (g) the majority of clients had a diagnosis of schizophrenia. The effect sizes were obtained by comparing the results on individual, standardized outcome measures 
from the treatment condition to those from the control condition at the end of treatment. Thus, effect sizes were only computed for studies with a control condition. Ranges for effect sizes were as follows: $\leq .2$ (minimal to small), .2-.5 (small to moderate), .5-.8 (moderate to large) and .8 and above (large) (Lipsey \& Wilson, 2001).

\subsection{Study outcome measures}

Outcome measures from the studies consisted of measures of social cognitive skills, cognitive skills, and measures of symptoms (positive, negative and general). We utilized conventions in the field of social cognitive research to group the social cognition measures into four major categories: emotion perception, social perception, theory of mind, and attributional style. For cognitive measures one interview-based measure of cognition was included as part of the aggregate cognition effect-size (Schizophrenia Cognition Rating Scale, SCRS, Keefe, Poe, Walker, Kang, \& Harvey, 2006). While we note that many clinician-rated scales of cognition in schizophrenia reported in the literature have only very weak relationships with performance-based measures of cognition, the SCRS has shown correlations of moderate-large magnitude $(r>.5)$ with well-accepted, comprehensive assessments of performance-based cognition in schizophrenia.

\subsection{Statistical analysis}

The effect-size analyses were conducted according to the procedures suggested by Rosenthal (1986) and Hedges and Olkin (1985). Comprehensive MetaAnalysis v. 2 (Borenstein, Hedges, Higgins, \& Rothstein, 2005) was used to calculate the effect size analyses. The dependent measures were organized into four categories to assess proximal effects and generalization of training to other illness features: (1) measures of social cognitive skills (proximal), (2) measures of positive, negative and general symptoms (generalization), and measures of cognition. The unit of analysis in a meta-analysis is the effect size (d). For purposes of the present study the $d$ score was defined as the difference between intervention type (i.e., treatment versus control) at termination of training expressed in standard deviation units ( $M_{\text {post exp. }}-M_{\text {post control }} / S D_{\text {pooled across groups }}$ ). Study statistics were converted to $d$ using the formulas provided by Glass (1977). We used the pooled standard deviation using the formula of Rosenthal (1986). Because of the potential for inflated within-group effects relative to between group comparisons, we did not compare within-group pre- to post-treatment change.

We predicted beneficial effects of social cognitive training on outcome measures in this paper. Thus, for studies with multiple measures in either the same social cognitive (facial affect recognition, ToM, or attributional style), symptom (positive, 
negative or general symptoms) or cognitive domain, we selected the measure within that domain with the middlemost-sized effect when the number of measures described in the study was odd, and the lower of the two middlemostsized effects when the number of measures described in the study was even. By expressing effect size in standard deviation units, we were able to make a direct comparison of outcomes across studies. The effects were categorized as small $(d<.4)$, moderate-large $(d=.4-.8)$ or large $(d>0.8$ or greater).

Positive effect size values indicated improvement as a result of social-cognitive interventions. Individual values of $d$ were thereafter combined across studies and weighted according to their precision. In this approach, larger sample-size, more precise (less variable estimates) is accorded a greater weight in the creation of the summary effect-size estimate. To partially address the "file-drawer" problem, we calculated a fail-safe $\mathrm{N}$ for each class of outcome variable by the method of Orwin (1983). This measure provides an estimate of the number of studies with null results that would be needed to render the effect size non-significant. In the absence of a universally accepted significance level for effect sizes, an effectsize of .20 would cease to reflect a meaningful degree of difference between treatment and control groups, as scores from $92 \%$ of participants from the two groups would overlap at this effect-size (Orwin, 1983). Lastly, we rated each study according to a 5-point study quality scale according to the following criteria: 1-point for use of randomization procedures, 1-point for description of fidelity maintenance, 1-point for blindedness of raters, 1-point for greater than 30 participants and 1-point for an active control condition in the study design. Interrater reliability for this study-quality scale was assessed by performing independent ratings of $25 \%$ of the studies included in this analysis by two of the co-authors (M.M.K. and E.G.). Reliability for these studies was .95.

\section{Results}

\subsection{Study characteristics}

A total of 16 studies were identified (see Table 3) with 15 reporting age of participants and 14 reporting gender. Eleven studies reported average years of education, and 13 studies reported average duration of illness. A detailed summary of the characteristics of these study samples is presented in Table 1. A total of seven at least somewhat separate models of comprehensive social cognitive treatment were identified and ordered according to the number of controlled studies investigating each program efficacy: Social Cognition and Interaction Training (SCIT; Penn, Roberts, Combs, \& Sterne, 2007; $k=6$ ), Social Cognitive Skills Training (SCST; Horan et al., 2009; $k=3$ ), Social Cognitive Training Program ( Gil-Sanz et al., 2009 and Gil-Sanz et al., 2014; $k=2$ ), Social 
Cognition and Enhancement Training (SCET; Choi \& Kwon, 2006; $k=1$ ), Instrumental Enrichment Program (IEP; Roncone et al., 2004; $k=1$ ), Metacognition and Social Cognitive Skills Training (MSCT; Rocha \& Queirós, 2013; $k=1$ ), Emotion and ToM Imitation Training (ETIT; Mazza et al., 2010; $k=1$ ); and a combined Emotion Perception and Theory-of-Mind videobased intervention (Bechi et al., 2012; $k=1$ ). Elements of one treatment model, Social Cognition and Interaction Training (SCIT), were included in one other treatment programs covered in this review: SCST. A summary of comprehensive social cognitive training models is presented in Table 2.

Table 1

Summary of sample characteristics for the social cognitive training group from the 16 studies included in the effect-size analysis (weighted means or proportions).

\begin{tabular}{lccc}
\hline & Mean or \% & N Studies & N Participants \\
\hline Participants & $100 \%$ & 16 & 313 \\
Age & 36.19 & 15 & 279 \\
Gender (\% males) & $64.92 \%$ & 14 & 263 \\
Education & 12.52 & 11 & 188 \\
IQ (estimate or full-scale) & 90.55 & 4 & 87 \\
Outpatient (\%) & 91.05 & 16 & 313 \\
Illness duration & 15.05 & 13 & 240 \\
Age of onset or first hospitalization & 22.39 & 13 & 240 \\
No. of hospitalizations & 4.28 & 4 & 92 \\
PANSS Positive & 16.16 & 8 & 168 \\
PANSS Negative & 18.91 & 9 & 186 \\
Chlorpromazine equivalent dose (mg/day) & 460.44 & 5 & 99 \\
\hline
\end{tabular}

\begin{tabular}{|c|c|c|c|}
\hline Treatment model & Areas of social cognition targeted & Methods of intervention & Duration and intensity \\
\hline $\begin{array}{l}\text { Social Cognition and Interaction Training } \\
\text { (Penn et al., 2007) }\end{array}$ & $\begin{array}{l}\text { Attributional biases, ToM and } \\
\text { emotion perception. }\end{array}$ & $\begin{array}{l}\text { Psychoeducation, drill-and-practice exercises, strategy games, } \\
\text { heuristic practice and role-plays. }\end{array}$ & 20-24 weekly sessions \\
\hline $\begin{array}{l}\text { Social Cognitive Skills Training } \\
\text { (Horan et al., 2009; Horan et al., 2011) }\end{array}$ & $\begin{array}{l}\text { Emotion perception, social } \\
\text { perception, ToM and attributional } \\
\text { bias }\end{array}$ & $\begin{array}{l}\text { Psychoeducation, drill-and-practice exercises, strategy games } \\
\text { and role plays. }\end{array}$ & 24 sessions over 12 weeks \\
\hline $\begin{array}{l}\text { Metacognitive and Social Cognitive Skills } \\
\text { Program (Rocha \& Queirós, 2013) }\end{array}$ & $\begin{array}{l}\text { Emotion perception, social } \\
\text { perception, ToM, attributional bias } \\
\text { and metacognitive capacities }\end{array}$ & $\begin{array}{l}\text { Psychoeducation, drill-and-practice exercises, mimicry, and a } \\
\text { large focus on training in identifying information processing } \\
\text { biases. }\end{array}$ & 18 sessions over 2 weeks \\
\hline $\begin{array}{l}\text { Social Cognitive Training Program } \\
\text { (Gil-Sanz et al., 2009; Gil-Sanz et al., } \\
\text { 2014) }\end{array}$ & $\begin{array}{l}\text { Facial affect recognition and social } \\
\text { perception }\end{array}$ & $\begin{array}{l}\text { Psychoeducation and drill-and-practice in the expression of } \\
\text { facial affect, and slides depicting social scenes in which the } \\
\text { participant must identify and interpret key social cues in the } \\
\text { slide and give the slide a label. }\end{array}$ & $\begin{array}{l}20 \text { sessions, } 2 \text { times per } \\
\text { week }\end{array}$ \\
\hline $\begin{array}{l}\text { Social Cognition Enhancement Training } \\
\text { (Choi \& Kwon, 2006) }\end{array}$ & Social perception and ToM & $\begin{array}{l}\text { Drill-and-practice in identifying social cues in four-panel } \\
\text { cartoons, organizing the four panels of cartoons into a coherent } \\
\text { story and then explaining that story to the group. }\end{array}$ & $\begin{array}{l}2 \text { times per week for } 26 \\
\text { weeks }\end{array}$ \\
\hline $\begin{array}{l}\text { Instrumental Enrichment Program } \\
\text { (Mazza et al., 2010) }\end{array}$ & Emotion perception and ToM & $\begin{array}{l}\text { Changing coping styles from passive to active through } \\
\text { "mediated learning". Psychoeducation, motivational } \\
\text { enhancements, role-plays and drill-and-practice training. }\end{array}$ & $\begin{array}{l}22 \text { one-hour-per-week } \\
\text { sessions }\end{array}$ \\
\hline $\begin{array}{l}\text { Theory of Mind and Emotion Processing } \\
\text { Training (Bechi et al., 2012) }\end{array}$ & Emotion perception and ToM & $\begin{array}{l}\text { Thirty-six short videos from international movies in which } \\
\text { training is offered for identifying basic emotions in } 24 \text { of the } \\
\text { clips and decoding beliefs, irony and intentions (ToM) in } 12 \text { of } \\
\text { the clips. }\end{array}$ & $\begin{array}{l}12 \text { one-hour-per-week } \\
\text { sessions. }\end{array}$ \\
\hline
\end{tabular}

\subsection{Effect-size analysis}

\subsubsection{Social cognitive measures}

The results of the effect-size analysis are presented in Table 4 Of the 16 controlled studies identified, 12 provided analyzable data on facial affect identification tasks that involved assigning descriptive labels to faces of different 
emotions (studies 1, 2, 3, 5, 7, 8, 9, 11, 12, 13, 15, 16). The weighted meaneffect-size was large $(d=.87)$ with a $95 \% \mathrm{Cl}$ of .46 to 1.29 . Because this $\mathrm{Cl}$ does not include 0 it can be considered to be statistically significant. Three studies provided information on facial affect discrimination in which faces are compared and a judgment is made regarding which face expresses a greater degree of emotion (studies $3,12,15$ ). The weighted mean effect size for these measures was non-significant.

\begin{tabular}{|c|c|c|c|c|c|c|c|c|}
\hline Study & Sample size & Population & $\begin{array}{l}\text { In/outpatient: } \\
\text { sample } \\
\text { characteristics }\end{array}$ & $\begin{array}{l}\text { Race/ethnicity } \\
\text { (\% White) }\end{array}$ & Age $(M, S D)$ & $\begin{array}{l}\text { Gender } \\
\text { ( }(\text { males) }\end{array}$ & Years of education (M, SD) & Duration of illness (years - M, SD) \\
\hline 1. Bechi et al. (2012) & $\begin{array}{l}\mathrm{SCT}=27 ; \\
\text { control }=24\end{array}$ & Schizophrenia & Outpatient & Not reported & $\begin{array}{l}\mathrm{SCT}=37.14(10.02) ; \\
\mathrm{TAU}=40.20(8.99)\end{array}$ & $\begin{array}{l}\mathrm{SCT}=68: \\
\mathrm{TAU}=67\end{array}$ & $\mathrm{SCT}=12.07$ (3.16); $\mathrm{TAU}=10.62,2.9$ & $\begin{array}{l}\mathrm{SCT}=14.00(9.08) ; \\
\mathrm{TAU}=16.62(6.40)\end{array}$ \\
\hline $\begin{array}{l}\text { 2. Choi and Kwon } \\
\text { (2006) }\end{array}$ & $\begin{array}{l}\text { SCET }=17 \\
\text { control }=17\end{array}$ & $\begin{array}{l}\text { Schizophrenia, } \\
\text { schizoaffective }\end{array}$ & Outpatient & Not reported & $\begin{array}{l}\text { SCET }=30.88(6.15) \text { : } \\
\text { control }=34.07(7.53)\end{array}$ & $\begin{array}{l}\text { SCET }=53 \\
\text { control }=59\end{array}$ & SCET $=13.06(2.22) ;$ control $=11.76(2.39)$ & $\begin{array}{l}\text { SCET = }=9.29(4.86) ; \\
\text { control }=13.08(6.29)\end{array}$ \\
\hline $\begin{array}{l}\text { 3. Combs, Adams, } \\
\text { et al. (2007) }\end{array}$ & $\begin{array}{l}\mathrm{SCrT}=18 ; \\
\text { control }=10\end{array}$ & $\begin{array}{l}\text { Schizophrenia } \\
\text { spectrum }\end{array}$ & Inpatient & $\begin{array}{l}\mathrm{SCIT}=61: \\
\text { control }=50\end{array}$ & $\begin{array}{l}\text { SCI }=41.3(11.2): \\
\text { control }=44(10.6)\end{array}$ & $\begin{array}{l}\mathrm{SCIT}=67: \\
\text { control }=90\end{array}$ & $\mathrm{SCIT}=11.3(1.6) ;$ control $=11.5(97)$ & $\begin{array}{l}\mathrm{SCT}=18.4(8.8): \\
\text { control }(19.7(75))\end{array}$ \\
\hline $\begin{array}{l}\text { 4. Gil-Sanz et al. } \\
(2009)\end{array}$ & $\begin{array}{l}\text { PECS }=7 \\
\text { control }=7\end{array}$ & Schizophrenia & Outpatient & Not reported & $\begin{array}{l}\text { PECS = 33.29 (8.36); } \\
\text { TAU = 41.43(9.03) }\end{array}$ & $\begin{array}{l}\mathrm{PECS}=57 \\
\mathrm{TAU}=43\end{array}$ & $\begin{array}{l}\text { \# completed high school: } \mathrm{PECS}=1 \text { : } \\
\text { TAU }=0\end{array}$ & $\mathrm{PECS}=13.43 ; \mathrm{TAU}=20.57$ \\
\hline $\begin{array}{l}\text { 5. Gil-Sanz et al. } \\
(2014)\end{array}$ & $\begin{array}{l}\mathrm{PECS}=20 \\
\text { control }=24\end{array}$ & Schizophrenia & Outpatient & Not reported & $\begin{array}{l}\text { PECS = } 37.05(6.43) \text { : } \\
\text { control }=43.83(9.77)\end{array}$ & $\begin{array}{l}\text { PECS }=40 \\
\text { control }=67\end{array}$ & $\begin{array}{l}\#(x)=0 \\
\text { TAU }=8(33 x)\end{array}$ & $\begin{array}{l}\text { PECS }=12.17(7.29): \\
\text { control }=16.22(10.59)\end{array}$ \\
\hline $\begin{array}{l}\text { 6. Gohar, Hamdi, El } \\
\text { Ray, Horan, and } \\
\text { Green (2013) }\end{array}$ & $\begin{array}{l}\mathrm{SCST}=22 ; \\
\text { control }=20\end{array}$ & $\begin{array}{l}\text { Schizophrenia, } \\
\text { schizoaffective }\end{array}$ & Outpatient & Not reported & $\begin{array}{l}\mathrm{SCST}=32.95(10.86): \\
\text { control }=30.75(10.58)\end{array}$ & $\begin{array}{l}\text { SCST }=73 ; \\
\text { control }=90\end{array}$ & SCST $=12.77(2.25) ;$ control $=12.05(1.88)$ & $\begin{array}{l}S \mathrm{SCT}=11 . .77(10.60): \\
\text { control }=8.40(7.02)\end{array}$ \\
\hline $\begin{array}{l}\text { 7. Hasson-Ohayon } \\
\text { et al (2014) }\end{array}$ & $\begin{array}{l}\mathrm{SCT}=34 ; \\
\text { control }=21\end{array}$ & SMI (sz, bipolar. & Outpatient & Not reported & $38.5(11.3)$ & 568 & 83\% completed high school & Not reported \\
\hline 8. Horan et al. (2009) & $\begin{array}{l}S \mathrm{SCST}=15 \\
\text { control }=16\end{array}$ & $\begin{array}{l}\text { Schizophrenia, } \\
\text { schizoaffective }\end{array}$ & Outpatient & $\begin{array}{l}\text { SCST }=27 ; \\
\text { control }=31\end{array}$ & $\begin{array}{l}\mathrm{SCST}=50.7(5.8): \\
\text { control }=45.9(7.5)\end{array}$ & $\begin{array}{l}\mathrm{SCST}=87 ; \\
\text { control }=100\end{array}$ & SCST $=12.5(1.1) ;$ control $=12.1(0.6)$ & $\begin{array}{l}S C S T=20.23(12.3) \\
\text { control }=18.03(7.4)\end{array}$ \\
\hline Study & Study design & $\begin{array}{l}\text { Dropout rate } \\
\text { ( } \mathbf{8} \text { groups attended) }\end{array}$ & $\begin{array}{l}\text { Treatment } \\
\text { condition }\end{array}$ & Control condition & $\begin{array}{l}\text { Social cognition outcome } \\
\text { measures }\end{array}$ & $\begin{array}{l}\text { Other } \\
\text { measures }\end{array}$ & Major findings & Rating \\
\hline 1. Bechi et al. (2012) & Quasi-experimental & Not reported & $\begin{array}{l}\text { Video-based } \\
\text { social cog } \\
\text { training (SCT) }\end{array}$ & $\begin{array}{l}\text { Integrated } \\
\text { Psychological Therapy } \\
\text { social cog training (SRT) } \\
\text { OR TAU }\end{array}$ & POFA, PST & PANSS, BACS & $\begin{array}{l}\text { ToM improved in SCT group relative to SRT } \\
\text { and control; no improvements on emotion } \\
\text { perception among groups }\end{array}$ & $\begin{array}{l}\text { Study quality rating of } 1: \text { greater } \\
\text { than } 30 \text { subjects }\end{array}$ \\
\hline $\begin{array}{l}\text { 2. Choi and Kwon } \\
\text { (2006) }\end{array}$ & RCT & $\begin{array}{l}\text { 41\% did not complete } \\
\text { all three levels of SCET }\end{array}$ & $\begin{array}{l}\text { Social } \\
\text { Cognition } \\
\text { Enhancement } \\
\text { Training } \\
\text { (SCET) }\end{array}$ & $\begin{array}{l}\text { Standard psychiatric } \\
\text { rehabilitation training }\end{array}$ & $\begin{array}{l}\text { WISC-R (picture arrangement), } \\
\text { ERT, SBST }\end{array}$ & None & $\begin{array}{l}\text { Sig improvement on WISC-R PA; improvement } \\
\text { on SBST at post-test, but not at follow up; no } \\
\text { improvement on ERT }\end{array}$ & $\begin{array}{l}\text { Study quality rating of } 3: \\
\text { randomizzation, greater than } 30 \\
\text { subjects, active control }\end{array}$ \\
\hline $\begin{array}{l}\text { 3. Combs, Adams, } \\
\text { et al. (2007) }\end{array}$ & Quasi-experimental & 96\% attendance rate & sar & Coping skills group & $\begin{array}{l}\text { FETr, FEDT, SPS, Hinting, AlHQ } \\
\text { NCS, TMT }\end{array}$ & $\begin{array}{l}\text { PANSS, WRAT, } \\
\text { Zigler-Glick } \\
\text { SFS }\end{array}$ & $\begin{array}{l}\text { Sig improvement on all social cog measures, } \\
\text { better self-reported social relationships, fewer } \\
\text { aggressive incidents on the unit }\end{array}$ & $\begin{array}{l}\text { Study quality rating of 2: blinded } \\
\text { raters, active control }\end{array}$ \\
\hline $\begin{array}{l}\text { 4. Gil-Sanz et al. } \\
\text { (2009) }\end{array}$ & RCT & Not reported & $\begin{array}{l}\text { Social } \\
\text { Cognition } \\
\text { Training } \\
\text { Program } \\
\text { (PECS) }\end{array}$ & TAU & SPS (translated), emotions task & $\begin{array}{l}\text { PANSS, } \\
\text { WHODAS, SP } \\
\text { scale }\end{array}$ & $\begin{array}{l}\text { Social perception improved, emotion } \\
\text { recognition did not }\end{array}$ & $\begin{array}{l}\text { Study quality rating of } 1 \text { : } \\
\text { randomization }\end{array}$ \\
\hline $\begin{array}{l}\text { 5. Gil-Sanz et al. } \\
\text { (2014) }\end{array}$ & RCT & Not reported & $\begin{array}{l}\text { Social } \\
\text { Cognition } \\
\text { Training } \\
\text { Program } \\
\text { (PECS) }\end{array}$ & $\begin{array}{l}\text { Psychiatric controls } \\
\text { received cog training. } \\
\text { healthy controls } \\
\text { received nothing }\end{array}$ & Hinting, emotions task & $\begin{array}{l}\text { PANSS, SP } \\
\text { scale }\end{array}$ & $\begin{array}{l}\text { Sg improvement in emotion recognition } \\
\text { and ToM }\end{array}$ & $\begin{array}{l}\text { Study quality rating of } 3 \text { : } \\
\text { randomization, blinded raters, } \\
\text { greater than } 30 \text { subjects }\end{array}$ \\
\hline 6. Gohar et al. (2013) & RCT & $\begin{array}{l}\mathrm{SCST}=13.55(2.13) \\
\text { control }=12.90 \\
(2.20)\end{array}$ & SCST & $\begin{array}{l}\text { Illness management } \\
\text { training }\end{array}$ & MSCER & $\begin{array}{l}\text { PANSS, } \\
\text { neurocog } \\
\text { battery }\end{array}$ & $\begin{array}{l}\text { Sig improvement in emotion perception and } \\
\text { managing emotions branches of MSCEIT }\end{array}$ & $\begin{array}{l}\text { Study quality rating of } 4: \\
\text { randomization, blinded raters, } \\
\text { greater than } 30 \text { subjects, active } \\
\text { control }\end{array}$ \\
\hline $\begin{array}{l}\text { 7. Hasson-Ohayon } \\
\text { et al. (2014) }\end{array}$ & RCT & $\begin{array}{l}\text { Percentage of sessions } \\
\text { attended }=71 \%\end{array}$ & $\begin{array}{l}\text { scri + social } \\
\text { mentoring }\end{array}$ & Social mentoring & FEIT, faux pas task, AIHQ & SPS & $\begin{array}{l}\text { Improvement in social engagement, TOM - FETT } \\
\text { improved but time x group interaction not sig }\end{array}$ & $\begin{array}{l}\text { Study quality rating of } 4 \text { : } \\
\text { randomization, fidelity maintenance, } \\
\text { greater than } 30 \text { subjects, active } \\
\text { control }\end{array}$ \\
\hline 8. Horan et al. (2009) & RCT & $\begin{array}{l}838 \text { mean session } \\
\text { attendance }\end{array}$ & SCST & $\begin{array}{l}\text { Illness } \\
\text { self-management and } \\
\text { relapse prevention }\end{array}$ & FEIT, PONS, AIHQ, TASTT & MCCB, BPRS & Sig improvement in facial affect perception & $\begin{array}{l}\text { Study quality rating of } 3: \\
\text { randomization, greater than } 30 \\
\text { subjects, active control }\end{array}$ \\
\hline
\end{tabular}




\begin{tabular}{|c|c|c|c|c|c|c|c|c|}
\hline & & & & skills training & & & & \\
\hline Study & Sample size & Population & $\begin{array}{l}\text { In/outpatient; } \\
\text { sample } \\
\text { characteristics }\end{array}$ & $\begin{array}{l}\text { Race/ethnicity } \\
\text { (\$ White) }\end{array}$ & Age (M, SD) & $\begin{array}{l}\text { Gender } \\
(\boldsymbol{\delta} \text { males })\end{array}$ & Years of education $(M, S D)$ & Duration of illness (years - M. SD) \\
\hline 9. Horan et al. (2011) & $\begin{array}{l}\text { SCST }=16 ; \\
\text { NR }=19 ; \\
\text { hybrid = 14; } \\
\text { ST }=19\end{array}$ & $\begin{array}{l}\text { Schizophrenia, } \\
\text { schizoaffective, } \\
\text { delusional disorder, } \\
\text { psychosis NOS }\end{array}$ & Outpatient & $\begin{array}{l}\mathrm{SCST}=25 ; \mathrm{NR}=21 \\
\text { hybrid }=36 ; \mathrm{ST}=37\end{array}$ & $\begin{array}{l}\mathrm{SCST}=51(7.1) ; \mathrm{NR}=46.6(7.4) \\
\text { hybrid }=50.4(10.1): \\
S T=45.1(11.2)\end{array}$ & $\begin{array}{l}\mathrm{SCST}=94 ; \\
\mathrm{NR}=89 \\
\text { hybrid }=93 \\
\mathrm{ST}=79\end{array}$ & $\begin{array}{l}\mathrm{SCST}=12.9(1.5) ; \mathrm{NR}=13.3(2.5) \\
\text { hybrid }=12.6(1.4) ; \mathrm{ST}=12.7(2.0)\end{array}$ & Reported age of onset, not duration \\
\hline $\begin{array}{l}\text { 10. Mazza et al. } \\
\text { (2010) }\end{array}$ & $\begin{array}{l}\text { EMTT }=16 ; \\
\text { control = } 17\end{array}$ & Schizophrenia & Outpatient & Not reported & $\begin{array}{l}\mathrm{ETTI}=24.37(2.12) \\
\text { control }=24.71(2.17)\end{array}$ & Not reported & $\mathrm{ETIT}=12.6(1.25) ;$ control $=10.3(2.57)$ & $\begin{array}{l}\text { Months of illness: ETTI = 6.3 (25): } \\
\text { control = } 6.5(4)\end{array}$ \\
\hline $\begin{array}{l}\text { 11. Roberts and Penn } \\
\text { (2009) }\end{array}$ & $\begin{array}{l}\mathrm{sCrT}=20 \\
\text { control }=11\end{array}$ & Schizophrenia & Outpatient & $\begin{array}{l}\mathrm{SCT}=75 \\
\text { control }=72.7\end{array}$ & $\begin{array}{l}\mathrm{SCT}=36.8(12.3) \\
\text { control }=41.4(12.3)\end{array}$ & $\begin{array}{l}\text { SCT }=55 \\
\text { control }=64\end{array}$ & $\mathrm{SCIT}=13.9(3.6) ;$ control $=14.0(1.8)$ & Not reported \\
\hline $\begin{array}{l}\text { 12. Roberts et al. } \\
\text { (2014) }\end{array}$ & $\begin{array}{l}\mathrm{SCT}=33: \\
\text { control }=33\end{array}$ & $\begin{array}{l}\text { Schizophrenia, } \\
\text { schizoaffective }\end{array}$ & Outpatient & $\begin{array}{l}\mathrm{SCT}=54.6: \\
\text { control }=72.7\end{array}$ & $\begin{array}{l}\mathrm{SCr}=40(12.2): \\
\text { control }=39.4(10.8)\end{array}$ & $\begin{array}{l}\mathrm{SCT}=66.7 \\
\text { control }=66.7\end{array}$ & $\begin{array}{l}\#(x) \text { completed high school: } \\
\text { scr }=22(66.6 \%) ; \text { control }=22(66.6 \%)\end{array}$ & $\begin{array}{l}\text { Age of first hosp: SCTT }=23.0 \\
(8.2) \text { : controls }=22.9(8.0)\end{array}$ \\
\hline $\begin{array}{l}\text { 13. Rocha and } \\
\text { Queirós (2013) }\end{array}$ & $\begin{array}{l}\text { MCST = 19; } \\
\text { control = } 16\end{array}$ & Schizophrenia & Outpatient & Not reported & $\begin{array}{l}\text { MCST }=38.63(8.88): \\
\text { control = 35.94 (8.69) }\end{array}$ & $\begin{array}{l}\text { MCST = } 84 \\
\text { control }=94\end{array}$ & $\begin{array}{l}\text { \% completed 9-12 years.: MCST }=53 ; \\
\text { control }=44\end{array}$ & $\begin{array}{l}\text { MCST }=13.68(7.49) \\
\text { control }=13.63(8.97)\end{array}$ \\
\hline $\begin{array}{l}\text { 14. Roncone et al. } \\
\text { (2004) }\end{array}$ & $\begin{array}{l}\text { MCSR }=10 \\
\text { control }=10\end{array}$ & Schizophrenia & Inpatient & Not reported & $\begin{array}{l}\text { MCSR }=33.9 \\
\text { control }=33.5\end{array}$ & $\begin{array}{l}\text { MCSR }=60 \\
\text { control }=70\end{array}$ & MCSR $=11.8(2.6) ;$ control $=11.1(2.8)$ & $\begin{array}{l}\text { MCSR }=16.9(8.05) \\
\text { control }=11.1(6.9)\end{array}$ \\
\hline $\begin{array}{l}\text { 15. Tas, Danaci, } \\
\text { Cubukcuoglu, and } \\
\text { Brüne (2012) }\end{array}$ & $\begin{array}{l}\text { SCIT }=19, \\
\text { control }=26\end{array}$ & Schizophrenia & Outpatient & Not reported & $\begin{array}{l}\mathrm{scr}=33.32(11.57), \\
\text { control }=34.62(10.6)\end{array}$ & $\begin{array}{l}\mathrm{sCr}=57.9, \\
\text { control }=46.2\end{array}$ & $\mathrm{SCCT}=11.95(1.72) ;$ control $=10.5(3.46)$ & $\begin{array}{l}\mathrm{SCTT}=12.63(9.99) ; \\
\text { control }=11.85(8.73)\end{array}$ \\
\hline $\begin{array}{l}\text { 16. Wang et al. } \\
\text { (2013) }\end{array}$ & $\begin{array}{l}\mathrm{SCIT}=22, \\
\text { control }=17\end{array}$ & Schizophrenia & Outpatient & Not reported & $\begin{array}{l}\text { SCT }=43.86(11.65) \\
\text { control }=40.88(10.15)\end{array}$ & $\begin{array}{l}\mathrm{SCT}=54.5 \\
\text { control }=47.1\end{array}$ & $\mathrm{SCCT}=10.10(2.14) ;$ control $=10.88(2.71)$ & Not reported \\
\hline Study & Study design & $\begin{array}{l}\text { Dropout rate ( }(\pi) \\
\text { groups attended) }\end{array}$ & $\begin{array}{l}\text { Treatment } \\
\text { condition }\end{array}$ & Control condition & $\begin{array}{l}\text { Social cognition outcome } \\
\text { measures }\end{array}$ & $\begin{array}{l}\text { Other } \\
\text { measures }\end{array}$ & Major findings & Ratings \\
\hline 9. Horan et al. (2011) & RCT & $\begin{array}{l}\text { SCST }=19.6(3.9) \\
\mathrm{NR}=19.3(39) \\
\text { hybrid }=20.1(2.7) \\
S T=20.1(2.7)\end{array}$ & SCST & $\begin{array}{l}\text { Standard illness } \\
\text { management skills } \\
\text { training }\end{array}$ & FET, MSCET, PONS, AlHQ TAST & BPRS, SANS & $\begin{array}{l}\text { SCST demonstrated greater improvements than } \\
\text { comparison groups in facial affect perception } \\
\text { and emotion management }\end{array}$ & $\begin{array}{l}\text { Study quality rating of } 4 \text { : } \\
\text { randomization, blinded raters, } \\
\text { greater than } 30 \text { subjects, active } \\
\text { control }\end{array}$ \\
\hline $\begin{array}{l}\text { 10. Mazza et al. } \\
(2010)\end{array}$ & RCT & No dropouts & $\begin{array}{l}\text { Emotion and } \\
\text { Theory of Mind } \\
\text { Imitation } \\
\text { Training (ETIT) }\end{array}$ & $\begin{array}{l}\text { Problem Solving Skills } \\
\text { Training (PST) }\end{array}$ & $\begin{array}{l}\text { Advanced Theory of Mind Scale, } \\
\text { Emotion Attribution Task, EQ }\end{array}$ & $\begin{array}{l}\text { BPRS, PSP, } \\
\text { neurocognitive } \\
\text { battery }\end{array}$ & $\begin{array}{l}\text { Sig improvement in emotion recognition and } \\
\text { ToM, also social functioning }\end{array}$ & $\begin{array}{l}\text { Study quality rating of } 3: \\
\text { randomization, greater than } 30 \\
\text { subjects, active control }\end{array}$ \\
\hline $\begin{array}{l}\text { 11. Roberts and Penn } \\
\text { (2009) }\end{array}$ & Quasi-experimental & $\begin{array}{l}\text { Intent to treat }=64 \% \text {, } \\
\text { completer }=82 \%\end{array}$ & scrt & TAU & FET, BLERT, Hinting, TAST, AIHQ & SSPA, PANSS & $\begin{array}{l}\text { Sig improvement in emotion perception and } \\
\text { social skill }\end{array}$ & $\begin{array}{l}\text { Study quality rating of } 1 \text { : greater } \\
\text { than } 30 \text { subjects }\end{array}$ \\
\hline $\begin{array}{l}12 \text { Roberts et al. } \\
\text { (2014) }\end{array}$ & RCT & $\begin{array}{l}\text { Average of 65\% } \\
\text { sessions attended }\end{array}$ & scr & TAU & $\begin{array}{l}\text { FET, FEDT, ER-4Q, Hinting, TAST, } \\
\text { AlHQ OSCARS }\end{array}$ & $\begin{array}{l}\text { GSFS, SSPA, } \\
\text { QOL, SCORS }\end{array}$ & $\begin{array}{l}\text { Improved social fx, neg sxs; AlHQ hostility - } \\
\text { trend level main effect for group, sig decrease } \\
\text { at follow up }\end{array}$ & $\begin{array}{l}\text { Study quality rating of } 4 \text { : } \\
\text { randomization, fidelity } \\
\text { maintenance, blinded raters, } \\
\text { greater than } 30 \text { subjects }\end{array}$ \\
\hline $\begin{array}{l}\text { 13. Rocha and } \\
\text { Queirós (2013) }\end{array}$ & Quasi-experimental & $\begin{array}{l}\text { MCST group attended } \\
\text { at least } 80 \% \text { of } \\
\text { sessions }\end{array}$ & MCST & TAU & $\begin{array}{l}\text { FET, MSCEr, Hinting AIHQ SPS, } \\
\text { fish task }\end{array}$ & $\begin{array}{l}\text { TMT, LSP. } \\
\text { PANSS }\end{array}$ & $\begin{array}{l}\text { Sig improvement in ToM, social perception, } \\
\text { emotion recognition, social functioning reduced } \\
\text { tendency to jump to conclusions }\end{array}$ & $\begin{array}{l}\text { Study quality rating of } 1 \text { : greater } \\
\text { than } 30 \text { subjects }\end{array}$ \\
\hline $\begin{array}{l}\text { 14. Roncone et al. } \\
\text { (2004) }\end{array}$ & RCT & Not reported & $\begin{array}{l}\text { Metacognitive } \\
\text { social } \\
\text { cognition } \\
\text { rehabilititation }\end{array}$ & 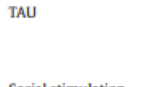 & $\begin{array}{l}\text { Tom stories, emotion recognition } \\
\text { task, Mach IN scale }\end{array}$ & $\begin{array}{l}\text { BPRS, verbal } \\
\text { fluency, wCST. } \\
\text { Tower of } \\
\text { London Test }\end{array}$ & $\begin{array}{l}\text { Sig improvement in ToM, emotion recognition, } \\
\text { and social functioning }\end{array}$ & $\begin{array}{l}\text { Study quality rating of } 1 \text { : } \\
\text { randomization }\end{array}$ \\
\hline 15. Tas et al. (2012) & RCT & Not reported & $\begin{array}{l}\text { Family-focused } \\
\text { Scrt }\end{array}$ & Social stimulation & $\begin{array}{l}\text { FEIT, FEDT, Hinting, Eyes, UOT, } \\
\text { IPSAQ }\end{array}$ & $\begin{array}{l}\text { BCIS, SFS, QLS, } \\
\text { PANSS }\end{array}$ & $\begin{array}{l}\text { Improved quality of life, social fx, symptoms, } \\
\text { ToM (Hinting). emotion perception, empathy } \\
\text { (UOT), self-certainty (BCS) }\end{array}$ & $\begin{array}{l}\text { Study quality rating of } 5 \text { : } \\
\text { randomization, fidelity } \\
\text { maintenance, blinded raters, } \\
\text { greater than } 30 \text { subjects, active } \\
\text { control }\end{array}$ \\
\hline $\begin{array}{l}\text { 16. Wang et al. } \\
\text { (2013) }\end{array}$ & Quasi-experimental & $\begin{array}{l}\text { None dropped from } \\
\text { SCrI group, did not } \\
\text { report \& sessions } \\
\text { attended }\end{array}$ & scr & TAU (wait-list control) & FErT, Eyes (Chinese version), ASQ & $\begin{array}{l}\text { PANSS, WAIS, } \\
\text { PSP }\end{array}$ & $\begin{array}{l}\text { Sig improvement in sodial fx, emotion perception } \\
\text { and ToM; re: attributions, no sig effect for mean } \\
\text { factor soores but sig effect for deviations to } \\
\text { neutral attributions }\end{array}$ & $\begin{array}{l}\text { Study quality rating of } 4 \text { : } \\
\text { randomization, fidelity } \\
\text { maintenance, blinded raters, } \\
\text { greater than } 30 \text { subjects }\end{array}$ \\
\hline
\end{tabular}

Table 4

Effect-size analysis of social cognitive, cognitive, symptoms and social functioning measures from integrated programs of social cognitive training $(k=16)$.

\begin{tabular}{|c|c|c|c|c|c|c|}
\hline & $k$ & $d$-Value & $95 \% \mathrm{Cl}$ & Z-statistic & p-Value & $\begin{array}{l}\text { Fail-safe } \\
\mathrm{N}\end{array}$ \\
\hline \multicolumn{7}{|l|}{ Social cognitive measures } \\
\hline Affect perception & & & & & & 38 \\
\hline $\begin{array}{l}\text { Facial affect } \\
\text { recognition }\end{array}$ & 12 & .84 & $.51 / .89$ & 7.16 & .000 & \\
\hline $\begin{array}{l}\text { Facial affect } \\
\text { discrimination }\end{array}$ & 3 & .90 & $-.37 / 2.17$ & 1.39 & .186 & \\
\hline Social perception & 4 & 1.29 & $.53 / 2.06$ & 3.30 & .001 & 22 \\
\hline Theory-of-mind & 13 & .70 & $.27 / 1.12$ & 3.23 & .001 & 18 \\
\hline \multicolumn{7}{|l|}{ Attributional style } \\
\hline Aggression & 6 & .30 & $.03 / .57$ & 2.21 & .027 & 3 \\
\hline Blame & 5 & .48 & $.08 / .87$ & 2.37 & .018 & 7 \\
\hline Hostility & 7 & .52 & $.10 / .93$ & 2.45 & .014 & 11 \\
\hline \multicolumn{7}{|l|}{ Symptoms } \\
\hline Positive symptoms & 7 & .27 & $-.07 / .61$ & 1.56 & .118 & \\
\hline Negative symptoms & 10 & .32 & $.01 / .63$ & 2.00 & .013 & 6 \\
\hline General symptoms & 4 & .40 & $.09 / .72$ & 2.53 & .011 & 4 \\
\hline \multicolumn{7}{|l|}{ Cognition } \\
\hline Summary measures & 3 & -.31 & $-.62 /-.01$ & -2.05 & .045 & 2 \\
\hline Executive function & 4 & 1.70 & $.18 / 3.23$ & 2.19 & .029 & 30 \\
\hline
\end{tabular}

Four studies included analyzable data on social perception measures (studies 2, $3,4,13)$. These measures all used social stimuli in which the participant was required to identify the nature of social interactions between people by verbal description or sequencing of stimuli. The weighted mean-effect-size from these studies was large ( $d=1.29, \mathrm{Cl}: 53 / 2.06)$. 
Thirteen studies included analyzable data on ToM measures (studies 1, 3, 5, 7, $8,9,10,11,12,13,14,15,16)$. These measures all included an assessment of a participant's ability to attribute accurate intentions, knowledge and emotions of individuals in specific social situations. The weighted mean effect-size for these studies was moderate-large ( $d=.70 ; \mathrm{Cl}: .27 / 1.12)$. Seven studies $(3,7,8,9,11$, $12,13)$ investigated the effects of social cognitive training on attributional style in schizophrenia all using the AIHQ in which participants read a series of vignettes describing a series of social situations and answered questions about the intentions of the character and how they themselves would respond in that situation. Weighted mean effect sizes were significantly different from 0 and small in size for aggression $(d=.30 ; \mathrm{Cl}: .03 / .57)$ and blame $(d=.48 ; \mathrm{Cl}: .08 / .87)$ and moderate in size for hostility $(d=.52 ; \mathrm{Cl}: .10 / .93)$.

\subsubsection{Symptoms}

The effects of comprehensive, social cognitive training programs on positive symptoms were not significant. The effects of social cognitive training programs on negative symptoms, evaluated in 10 studies $(3,4,6,8,9,10,12,13,14,15)$, were significant and small-to-moderate in size $(d=.32 ; \mathrm{Cl}$ : .01/.63) and the effects on general symptoms $(d=.40 ; \mathrm{Cl}: .09 / .72)$ were small-to-moderate, evaluated in 4 studies $(4,11,12,15)$.

\subsubsection{Cognition}

Significant, but small-sized negative effects $(d=-.31$; Cl: $-.62 /-.01)$ of social cognitive training programs were evident on summary measures of cognition which included performance or reports of function across a broad array of specific neurocognitive domains (studies 8, 9, 12). Social cognitive training studies reported large effect-size improvements $(d=1.70 ; \mathrm{Cl}$ : .18/3.23) on measures of executive-function which included skills such as planning and shifting set (studies $3,10,13,14)$.

\section{Discussion}

The results of this critical review and effect-size analysis of controlled, comprehensive social cognitive training programs revealed several interesting findings. First, seven different models of comprehensive training have been assessed in a controlled experimental design in a total of 16 studies. These programs differed on key characteristics such as duration of training (2.56 months), size of groups (3-12 members), and the number and type of social cognitive areas trained (2-4 areas). The most common duration of treatment in these studies was 6 months $(k=8)$, and the most common number of social 
cognitive areas treated in this corpus of studies was $3(k=8)$. Second, of the seven models, only three have been studied in more than a single study: Social Cognition and Interaction Training (SCIT; 6 controlled studies), Social Cognitive Skills Training (SCST; 3 controlled studies) and the Social Cognitive Training Program (2 controlled studies). Third, and most importantly, analysis of the results of individual studies revealed positive effects of social cognitive training in all 16 studies on a variety of proximal social cognitive measures. More specifically, $11 / 15$ controlled studies that measured facial affect recognition skills reported positive effects while 10/13 studies that included measures of theory-ofmind reported positive effects. In contrast, treatment effects on attributional style were evident in less than half the reports that studied this social cognitive domain (4/9). The number of studies including measures of social perception was very small but results from studies that included these measures were uniformly positive (4/4).

These latter observations were largely supported by the results of a formal effectsize analysis; measures of social cognition in aggregate revealed large effects of these social cognitive training programs on proximal measures of facial affect identification, social perception and theory-of-mind, and small-to-moderate sized effects on different aspects of attributional style. Modest but significant effects were also evident on more distal measures of general and negative symptoms not directly trained in these programs. The strength of the results from the literature review and effect-size analysis was bolstered by three methodological characteristics of this group of largely pilot studies: 13/16 studies were randomized, 12/16 studies included 30 or more participants and 11/16 studies used active control comparison groups. Thus, these findings provide clear evidence that the study of social cognitive training programs for schizophrenia is a fruitful line for further investigation with the potential for remediation of a key, historically treatment-resistant feature of the illness tied closely to functional outcome.

Importantly, these conclusions build on previous narrative and meta-analytic reviews, by excluding: (1) "proof-of-concept" studies consisting very brief (often one- or two-session) interventions that would be unlikely to produce sustained change in social cognitive skills in schizophrenia, and (2) studies including blended social cognitive and elementary cognitive training or other psychosocial training, making it easier to discern the specific effects of social cognitive training on any observed social cognitive change in these studies. For example, in the Kurtz and Richardson meta-analysis nearly $40 \%$ of included studies were either "proof of concept" or blended interventions. More generally, these findings support the conclusions of these previous reviews and provide additional 
evidence that a research base is developing that may support the inclusion of comprehensive social cognitive treatment programs for consideration as a novel and vital evidence-based practice to be added to the armamentarium of psychosocial treatments for people with schizophrenia in the not-too-distant future.

This narrative review and effect size analysis revealed that improvements in summary indices of elementary cognitive function did not accompany the effects of social cognitive training programs on proximal social cognitive outcome measures. This finding suggests that these social cognitive training programs do not exert their effects on social cognitive outcomes indirectly by improving overall levels of cognitive skill. Indeed, effects of these training programs on summary cognitive skill measures were negative, suggesting that these treatments may have worsened cognition. The small number of studies included in this analysis $(k=3)$, along with the borderline level of significance of this finding, encourages caution regarding its interpretation.

It is important to note that substantial improvements were evident in one specific domain of cognition: executive function (the skills in forming mental sets and being cognitively flexible) and these improvements could account for at least some of the changes in more complex social cognitive skills studied in this review. This possibility clearly merits further investigation. However, these conclusions too must be interpreted very cautiously in light of the small number of studies supporting them $(k=4)$.

The current findings regarding proximal social cognitive outcomes (e.g., affect recognition, ToM, attributional bias) are largely consonant with the findings of Kurtz and Richardson (2012) and in some cases provide even stronger evidence for the effectiveness of social cognitive training programs for these proximal outcome measures (e.g., affect recognition: $d=.71$ in Kurtz and Richardson (2012) vs. $d=.84$ in the current study; ToM: $d=.46$ in the previous analysis vs. $d=.70$ in the current analysis; significant treatment effects on attributional bias were evident in the current study but not in the previous one). The studies in this review have a number of limitations. First, only $7 / 16$ studies reviewed included blinded outcome raters. In light of the well-documented role of this design feature in inflating reported effect-sizes for another psychosocial treatment for schizophrenia, Cognitive Behavioral Therapy (Wykes, Steele, Everitt, \& Tarrier, 2008), caution regarding these reported results is warranted until further studies including blinded raters are conducted. Second, only 4/16 studies reported any type of methodology for maintaining treatment fidelity. Future research will need to ensure that these novel approaches to treating social cognitive deficits are being conducted according to the principles of treatment 
outlined by the authors of these interventions. Third, only two studies investigated durability of treatment effects (Combs et al., 2007,Combs et al., 2009 and Roberts et al., 2014). While results from these studies have been promising, the durability of social cognitive training treatment effects remains largely unknown. Fourth, the range of approaches to social cognitive training reviewed in this paper $(n=7)$, targeting different aspects of social cognition, and consisting of different therapeutic features in different quantities and combinations (e.g., role-plays, heuristic practice, modeling and drill-and-practice) underscores the absence of knowledge regarding the active ingredients of these complex interventions. Fifth, the psychometric characteristics of many of the social cognitive outcome measures selected for studies reviewed in this paper remain unclear, with floor and ceiling effects, and measures of reliability and sensitivity to change largely undocumented (Green et al., 2008 and Pinkham et al., 2014). These features of studied measures may have impacted the results of our review. Sixth, it is not impossible that the 6 studies selecting TAU as a control condition may have inflated observed effect-sizes. An analysis of results from studies of emotion identification however argues against this possibility: studies that selected an active control condition surprisingly reported larger effects of comprehensive social cognitive training programs on emotion identification skills than those that selected TAU as a control condition ( $d=1.07, k=7$, vs. $d=.55, k=5$ ).

Several limitations of the review should be noted. First, our exclusion criteria, which were selected to provide a unique assessment of the evidence-base for comprehensive social cognitive training programs, prevented us from evaluating the effects of social cognitive training programs when offered in concert with other forms of psychosocial rehabilitation. Second, the study of social cognitive training programs, while conceptualized as a distinct behavioral treatment approach in this review, contains many elements of other, evidence-based treatments (drilland-practice exercises from cognitive remediation, social role-plays with corrective feedback in social skills training). Thus by their very nature, social cognitive training programs overlap to some degree with other treatment modalities. Third, the relationship of different domains of social cognition to one another and their response to treatment remain unclear; while our review was restricted to interventions targeting two or more social cognitive domains, these interventions might still have impacted only one outcome social cognitive domain. Likewise, studies excluded from our review that targeted a single treatment in a single social cognitive domain may influence multiple social cognitive outcome domains. Fourth, significant effects of comprehensive social cognitive training programs on negative symptoms and summary measures of cognition were very modest (95\% Cl included .01 in both cases) and should be interpreted cautiously. 
Fifth, forming conclusions on the effects of these training programs on attributional bias remains difficult as some studies included in the review failed to show baseline differences on these measures between healthy people and people with schizophrenia (e.g., Horan et al., 2011). Fifth, we did not account for baseline differences on social cognitive or other outcome measures in our effectsize analysis. Nonetheless, of 11 studies that reported statistical comparisons between treatment and control groups on social cognitive measures before treatment, 8 failed to report statistical differences on any of the social cognitive measures reported in their study.

In summary, a critical literature review and effect-size (ES) analysis was conducted to investigate the efficacy of comprehensive programs of social cognitive training in schizophrenia. Results revealed 16 controlled studies consisting of seven models of comprehensive treatment with only three of these treatment models investigated in more than one study. The effects of social cognitive training were reported in 11/15 studies that included facial affect recognition skills ( $E S=.84$ ) and $10 / 13$ studies that included theory-of-mind $(E S=.70)$ as outcomes. Less than half $(4 / 9)$ of studies that measured attributional style as an outcome reported effects of treatment, but effect sizes across studies were significant (ESs $=.30-.52$ ). The effect sizes for symptoms were modest, but, with the exception of positive symptoms, significant (ESs $=.32-.40$ ).

\section{Funding source}

The funding sources for this study had no role in the study design, collection, analysis or interpretation of the data, the writing of the report and in the decisions to submit the manuscript for publication.

\section{Contributors}

Matthew M. Kurtz, Ph.D., was responsible for initial conceptualization of the current review (along with D.L.P.), oversaw article collection and analysis, formulated and conducted the effect-size analysis and wrote the first draft of the manuscript.

Emily Gagen helped with article searches, constructed descriptive tables in the manuscript, double-checked coding of design quality of studies and established reliability of coding with MMK, and edited the final manuscript.

Nuno Rocha, Ph.D., helped with article searches, constructed descriptive tables in the manuscript and edited the final manuscript.

Sergio Machado, Ph.D., helped with article searches and edited the final manuscript. 
David L. Penn, Ph.D., helped conceptualize the scope and focus of the analysis, and provided detailed edits on a previous draft of the manuscript.

Conflict of interest

The authors have no conflicts of interest to report.

Appendix A. Measures used in the effect-size analysis

A.1. Facial affect identification

Emotion recognition.

Emotion Recognition Test (Lee, 2001).

Face Emotion Identification Test (Kerr \& Neale, 1993).

NimStim Face Stimulus Set (Tottenham et al., 2009).

Pictures of Facial Affect (Ekman \& Friesen, 1976).

Emotion discrimination.

Face Emotion Discrimination Test (Kerr \& Neale, 1993).

\section{A.2. Social perception}

Social Perception Scale (García, 2003).

Picture Arrangement subtest of the WISC-R (Kaufman, 1979).

\section{A.3. Theory of mind}

Advanced Theory of Mind Scale (Blair \& Cipolotti, 2000).

Hinting Task (Corcoran, Mercer, \& Frith, 1995).

The Awareness of Social Inference Test (McDonald, Flanagan, Rollins, \& Kinch, 2003).

The Eyes Test (Baron-Cohen, Wheelwright, Hill, Raste, \& Plumb, 2001).

Theory of Mind Picture Sequencing Test (Brüne, 2003).

Faux Pas Task (Stone, Baron-Cohen, \& Knight, 1998).

\section{A.4. Attributional style}

Ambiguous Intentions Hostility Questionnaire (AIHQ; Combs, Penn, Wicher, \& Waldheter, 2007).

\section{A.5. Symptoms}

Positive and Negative Syndrome Scale (Kay, Flszbein, \& Opfer, 1987).

Brief Psychiatric Rating Scale (Ventura et al., 1993).
A.6. Cognition 


\section{A.6.1. Summary measures}

Matrics Consensus Cognitive Battery - Composite (Nuechterlein et al., 2008).

Schizophrenia Cognition Rating Scale - Total (Keefe et al., 2006).

\section{A.6.2. Executive function}

Tower of London (Shallice, 1982).

Trailmaking Test B (Reitan, 1992).

Wisconsin Card Sorting Test (Heaton, 1981).

\section{References}

Adolphs, 2009

R. Adolphs

The social brain: Neural basis of social knowledge

Annual Review of Psychology, 60 (2009), p. 693

American Psychiatric Association, 1987

American Psychiatric Association

Diagnostic and statistical manual of mental disorders

(3rd ed.) (1987) (rev.). Washington, DC)

Baron-Cohen et al., 2001

S. Baron-Cohen, S. Wheelwright, J. Hill, Y. Raste, I. Plumb

The "reading the mind in the eyes" test revised version: A study with normal adults, and adults with Asperger syndrome or high-functioning autism Journal of Child Psychology and Psychiatry, 42 (2) (2001), pp. 241-251

Bechi et al., 2012

M. Bechi, R. Riccaboni, S. Ali, F. Fresi, M. Buonocore, M. Bosia, ... R. Cavallaro Theory of mind and emotion processing training for patients with schizophrenia: Preliminary findings

Psychiatry Research, 198 (3) (2012), pp. 371-377

Blair and Cipolotti, 2000

R.J.R. Blair, L. Cipolotti 
Impaired social response reversal: A case of acquired sociopathy

Brain, 123 (6) (2000), pp. 1122-1141

Borenstein et al., 2005

M. Borenstein, L. Hedges., J. Higgins, H. Rothstein

Comprehensive meta-analysis version 2

Biostat, Engelwood, NJ (2005)

Brenner et al., 1994

H.D. Brenner, V. Roder, B. Hodel, N. Kienzle, D. Reed, R.P. Liberman

Integrated psychological therapy for schizophrenic patients (IPT)

Hogrefe \& Huber Publishers (1994)

Brüne, 2003

M. Brüne

Theory of mind and the role of IQ in chronic disorganized schizophrenia

Schizophrenia Research, 60 (1) (2003), pp. 57-64

Choi et al., 2009

J.H. Choi, J.H. Kim, J. Lee, M.F. Green

Social cognition training for individuals with schizophrenia: A review of targeted interventions

Clinical Psychopharmacological Neuroscience, 7 (2009), pp. 29-38

Choi and Kwon, 2006

K.H. Choi, J.H. Kwon

Social cognition enhancement training for schizophrenia: A preliminary randomized controlled trial

Community Mental Health Journal, 42 (2) (2006), pp. 177-187

Combs et al., 2007

D.R. Combs, S.D. Adams, D.L. Penn, D. Roberts, J. Tiegreen, P. Stem

Social Cognition and Interaction Training (SCIT) for inpatients with schizophrenia spectrum disorders: Preliminary findings 
Schizophrenia Research, 91 (1) (2007), pp. 112-116

Combs et al., 2009

D.R. Combs, K. Elerson, D.L. Penn, J.A. Tiegreen, A. Nelson, S.N. Ledet, et al.

Stability and generalization of Social Cognition Interaction Training (SCIT) for schizophrenia: Six-month follow-up results

Schizophrenia Research, 112 (1-3) (2009), pp. 196-197

Combs et al., 2007

D.R. Combs, D.L. Penn, M. Wicher, E. Waldheter

The Ambiguous Intentions Hostility Questionnaire (AIHQ): A new measure for evaluating hostile social-cognitive biases in paranoia

Cognitive Neuropsychiatry, 12 (2) (2007), pp. 128-143

Corcoran et al., 1995

R. Corcoran, G. Mercer, C.D. Frith

Schizophrenia, symptomatology and social inference: Investigating "theory of mind" in people with schizophrenia

Schizophrenia Research, 17 (1) (1995), pp. 5-13

Couture et al., 2006

S.M. Couture, D.L. Penn, D.L. Roberts

The functional significance of social cognition in schizophrenia: A review

Schizophrenia Bulletin, 32 (Suppl. 1) (2006), pp. S44-S63

Edmondson et al., 2012

M. Edmondson, R. Pahwa, K.K. Lee, M. Hoe, J.S. Brekke

A dual change model of life satisfaction and functioning for individuals with schizophrenia

Schizophrenia Research, 139 (1) (2012), pp. 110-115

Ekman and Friesen, 1976

P. Ekman, W.V. Friesen

Measuring facial movement 
Environmental Psychology \& Nonverbal Behavior, 1 (1) (1976), pp. 56-75

Fett et al., 2011

A.K.J. Fett, W. Viechtbauer, D.L. Penn, J. van Os, L. Krabbendam

The relationship between neurocognition and social cognition with functional outcomes in schizophrenia: A meta-analysis

Neuroscience \& Biobehavioral Reviews, 35 (3) (2011), pp. 573-588

Fiske, 1991

S.T. Fiske, S.E. Taylor

Social cognition

(2nd ed.)McGraw-Hill, New York, NY (1991)

Fiszdon and Reddy, 2012

J.M. Fiszdon, L.F. Reddy

Review of social cognitive treatments for psychosis

Clinical Psychology Review, 32 (8) (2012), pp. 724-740

García, 2003

S. García

Rehabilitación de enfermos mentales crónicos: Entrenamiento en Percepción Social

Trabajo de Investigación. Departamento de Personalidad, Evaluación y Tratamientos Psicológicos, Universidad de Valencia, Valencia, VLC (2003)

Gil-Sanz et al., 2014

D. Gil-Sanz, M. Fernández-Modamio, R. Bengochea-Seco, M. Arrieta-Rodríguez, G. Pérez-Fuentes

Efficacy of the Social Cognition Training Program in a sample of schizophrenic outpatients

Clinical Schizophrenia \& Related Psychoses, 1-27 (2014)

Gil-Sanz et al., 2009

D. Gil-Sanz, M.D. Lorenzo, R.B. Seco, M.A. Rodríguez, I.L. Martínez, R.S. Calleja, A.Á. Soltero 
Efficacy of a social cognition training program for schizophrenic patients: A pilot study

The Spanish Journal of Psychology, 12 (1) (2009), pp. 184-191

Glass, 1977

G.V. Glass

Integrating findings: The meta-analysis of research

Review of Research in Education, 351-379 (1977)

Gohar et al., 2013

S.M. Gohar, E. Hamdi, L. El Ray, W.P. Horan, M.F. Green

Adapting and evaluating a social cognitive remediation program for schizophrenia in Arabic

Schizophrenia Research, 148 (1) (2013), pp. 12-17

Green et al., 2008

M.F. Green, D.L. Penn, R. Bentall, W.T. Carpenter, W. Gaebel, R.C. Gur, ... R. Heinssen

Social cognition in schizophrenia: An NIMH workshop on definitions, assessment, and research opportunities

Schizophrenia Bulletin, 34 (6) (2008), pp. 1211-1220

Harvey et al., 2006

P.D. Harvey, T.L. Patterson, L.S. Potter, K. Zhong, M. Brecher

Improvement in social competence with short-term atypical antipsychotic treatment: A randomized, double-blind comparison of quetiapine versus risperidone for social competence, social cognition, and neuropsychological functioning

American Journal of Psychiatry, 163 (2006), pp. 1918-1925

Hasson-Ohayon et al., 2014

I. Hasson-Ohayon, M. Mashiach-Eizenberg, M. Avidan, D. Roberts, D. Roe

Social Cognition and Interaction Training: Preliminary results of an RCT in a community setting in Israel

Psychiatric Services, 65 (4) (2014), pp. 555-558 
Heaton, 1981

R.K. Heaton

A manual for the Wisconsin Card Sorting Test

Western Psychological Services (1981)

Hedges and Olkin, 1985

L.V. Hedges, I. Olkin

Statistical methods for meta-analysis

Academic Press, New York, NY (1985)

Hogarty et al., 2004

G.E. Hogarty, S. Flesher, R. Ulrich, M. Carter, D. Greenwald, M. Pogue-Geile, ... R. Zoretich

Cognitive enhancement therapy for schizophrenia: Effects of a 2-year randomized trial on cognition and behavior

Archives of General Psychiatry, 61 (9) (2004), pp. 866-876

Hogarty et al., 2006

G.E. Hogarty, D.P. Greenwald, S.M. Eack

Special section: A memorial tribute: Durability and mechanism of effects of cognitive enhancement therapy

Psychiatric Services, 57 (12) (2006), pp. 1751-1757

Horan et al., 2008

W.P. Horan, R.S. Kern, M.F. Green, D.L. Penn

Social cognition training for individuals with schizophrenia: Emerging evidence

American Journal of Psychiatric Rehabilitation, 11 (3) (2008), pp. 205-252

Horan et al., 2009

W.P. Horan, R.S. Kern, K. Shokat-Fadai, M.J. Sergi, J.K. Wynn, M.F. Green

Social cognitive skills training in schizophrenia: An initial efficacy study of stabilized outpatients 
Schizophrenia Research, 107 (1) (2009), pp. 47-54

Horan et al., 2011

W.P. Horan, R.S. Kern, C. Tripp, G. Helleman, J.K. Wynn, M.D. Bell, ... M.F. Green

Efficacy and specificity of social cognitive skills training for outpatients with psychotic disorders

Journal of Psychiatry Research, 45 (2011), pp. 1113-1122

Kaufman, 1979

A.S. Kaufman

Intelligent testing with the WISC-R

John Wiley \& Sons, New York (1979)

Kay et al., 1987

S.R. Kay, A. Flszbein, L.A. Opfer

The positive and negative syndrome scale (PANSS) for schizophrenia

Schizophrenia Bulletin, 13 (2) (1987), p. 261

Keefe et al., 2006

R.S. Keefe, M. Poe, T.M. Walker, J.W. Kang, P.D. Harvey

The Schizophrenia Cognition Rating Scale: An interview-based assessment and its relationship to cognition, real-world functioning, and functional capacity

American Journal of Psychiatry, 163 (2006), pp. 426-432

Kerr and Neale, 1993

S.L. Kerr, J.M. Neale

Emotion perception in schizophrenia: Specific deficit or further evidence of generalized poor performance?

Journal of Abnormal Psychology, 102 (2) (1993), p. 312

Kurtz and Richardson, 2012

M.M. Kurtz, C.L. Richardson

Social cognitive training for schizophrenia: A meta-analytic investigation of controlled research 
Schizophrenia Bulletin, 38 (5) (2012), pp. 1092-1104

Lee, 2001

S.J. Lee

Development of an emotional awareness test consisting of problem solving tasks

Korean Journal of Social Personality Psychology, 15 (3) (2001), pp. 65-86

Lipsey and Wilson, 2001

M.W. Lipsey, D.B. Wilson

The way in which intervention studies have "personality" and why it is important to meta-analysis

Evaluation \& the Health Professions, 24 (3) (2001), pp. 236-254

Mancuso et al., 2011

F. Mancuso, W.P. Horan, R.S. Kern, M.F. Green

Social cognition in psychosis: Multidimensional structure, clinical correlates, and relationship with functional outcome

Schizophrenia Research, 125 (2) (2011), pp. 143-151

Mazza et al., 2010

M. Mazza, G. Lucci, F. Pacitti, M.C. Pino, M. Mariano, M. Casacchia, R. Roncone

Could schizophrenic subjects improve their social cognition abilities only with observation and imitation of social situations?

Neuropsychological Rehabilitation, 20 (5) (2010), pp. 675-703

McDonald et al., 2003

S. McDonald, S. Flanagan, J. Rollins, J. Kinch

TASIT: A new clinical tool for assessing social perception after traumatic brain injury

The Journal of Head Trauma Rehabilitation, 18 (3) (2003), pp. 219-238

Nuechterlein et al., 2004

K.H. Nuechterlein, D.M. Barch, J.M. Gold, T.E. Goldberg, M.F. Green, R.K. Heaton

Identification of separable cognitive factors in schizophrenia 
Schizophrenia Research, 72 (1) (2004), pp. 29-39

Nuechterlein et al., 2008

K.H. Nuechterlein, M.F. Green, R.S. Kern, L.E. Baade, D.M. Barch, J.D. Cohen, ... S.R. Marder

The MATRICS Consensus Cognitive Battery, part 1: Test selection, reliability, and validity

American Journal of Psychiatry, 165 (2) (2008), pp. 203-213

Orwin, 1983

R.G. Orwin

A fail-safe $\mathbf{N}$ for effect size in meta-analysis

Journal of Educational Statistics, 157-159 (1983)

Penn et al., 2007

D.L. Penn, D.L. Roberts, D. Combs, A. Sterne

Best practices: The development of the Social Cognition and Interaction Training program for schizophrenia spectrum disorders

Psychiatric Services, 58 (4) (2007), pp. 449-451

Penn et al., 2008

D.L. Penn, L.J. Sanna, D.L. Roberts

Social cognition in schizophrenia: An overview

Schizophrenia Bulletin, 34 (3) (2008), pp. 408-411

Pinkham et al., 2014

A.E. Pinkham, D.L. Penn, M.F. Green, B. Buck, K. Healey, P.D. Harvey

The social cognition psychometric evaluation study: Results of the expert survey and RAND panel

Schizophrenia Bulletin, 40 (4) (2014), pp. 813-823

Pinkham et al., in press

A.E. Pinkham, D.L. Penn, M.F. Green, P.D. Harvey

Social cognition psychometric evaluation (SCOPE): Results of the initial psychometric study 
Schizophrenia Bulletin (2015) (in press)

Reitan, 1992

R.M. Reitan

Trail Making Test: Manual for administration and scoring

Reitan Neuropsychology Laboratory, Tuscon, AZ (1992)

Roberts et al., 2014

D.L. Roberts, D.R. Combs, M. Willoughby, J. Mintz, C. Gibson, B. Rupp, D.L. Penn A randomized, controlled trial of Social Cognition and Interaction Training (SCIT) for outpatients with schizophrenia spectrum disorders

British Journal of Clinical Psychology, 53 (3) (2014), pp. 281-298

Roberts and Penn, 2009

D.L. Roberts, D.L. Penn

Social Cognition and Interaction Training (SCIT) for outpatients with schizophrenia: A preliminary study

Psychiatry Research, 166 (2) (2009), pp. 141-147

Robertson et al., 2014

B.R. Robertson, D. Prestia, E.W. Twamley, T.L. Patterson, C.R. Bowie, P.D. Harvey Social competence versus negative symptoms as predictors of real world social functioning in schizophrenia

Schizophrenia Research, 160 (1) (2014), pp. 136-141

Rocha and Queirós, 2013

N.B. Rocha, C. Queirós

Metacognitive and social cognition training (MSCT) in schizophrenia: A preliminary efficacy study

Schizophrenia Research, 150 (1) (2013), pp. 64-68

Roder et al., 2006

V. Roder, D.R. Mueller, K.T. Mueser, H.D. Brenner

Integrated psychological therapy (IPT) for schizophrenia: Is it effective? 
Schizophrenia Bulletin, 32 (Suppl. 1) (2006), pp. S81-S93

Roder et al., 2011

V. Roder, D. Mueller, S. Schmidt

Effectiveness of integrated psychological therapy (IPT) for schizophrenia patients: a research update

Schizophrenia Bulletin, 37 (S2) (2011), pp. S71-S79

Roncone et al., 2004

R. Roncone, M. Mazza, I. Frangou, A. De Risio, D. Ussorio, C. Tozzini, M. Casacchia Rehabilitation of theory of mind deficit in schizophrenia: A pilot study of metacognitive strategies in group treatment

Neuropsychological Rehabilitation, 14 (4) (2004), pp. 421-435

Rosenthal, 1986

R. Rosenthal

Meta-analytic procedures for social research

Sage, London, UK (1986)

Savla et al., 2013

G.N. Savla, L. Vella, C.C. Armstrong, D.L. Penn, E.W. Twamley

Deficits in domains of social cognition in schizophrenia: A meta-analysis of the empirical evidence

Schizophrenia Bulletin, 39 (5) (2013), pp. 979-992

Schmidt et al., 2011

S.J. Schmidt, D.R. Mueller, V. Roder

Social cognition as a mediator variable between neurocognition and functional outcome in schizophrenia: Empirical review and new results by structural equation modeling

Schizophrenia Bulletin, 37 (Suppl. 2) (2011), pp. S41-S54

Shallice, 1982

T. Shallice

Specific impairments of planning 
Philosophical Transactions of the Royal Society B, 298 (1982), pp. 199-209

Stone et al., 1998

V.E. Stone, S. Baron-Cohen, R.T. Knight

Frontal lobe contributions to theory of mind

Journal of Cognitive Neuroscience, 10 (5) (1998), pp. 640-656

Tas et al., 2012

C. Tas, A.E. Danaci, Z. Cubukcuoglu, M. Brüne

Impact of family involvement on social cognition training in clinically stable outpatients with schizophrenia-A randomized pilot study

Psychiatry Research, 195 (1) (2012), pp. 32-38

Tottenham et al., 2009

N. Tottenham, J.W. Tanaka, A.C. Leon, T. McCarry, M. Nurse, T.A. Hare, ... C. Nelson

The NimStim set of facial expressions: Judgments from untrained research participants

Psychiatry Research, 168 (3) (2009), pp. 242-249

National Library of Medicine, 1994

US National Library of Medicine

National Institutes of Health

PubMed (1994)

Ventura et al., 1993

J. Ventura, D. Lukoff, K.H. Nuechterlein, R.P. Liberman, M.F. Green, A. Shaner

Manual for the expanded brief psychiatric rating scale

International Journal of Methods in Psychiatry Research, 3 (1993), pp. 221-224

Wang et al., 2013

Y. Wang, D.L. Roberts, B. Xu, R. Cao, M. Yan, Q. Jiang

Social Cognition and Interaction Training for patients with stable schizophrenia in Chinese community settings 
Psychiatry Research, 210 (3) (2013), pp. 751-755

Wolwer et al., 2010

W. Wolwer, D.R. Combs, N. Frommann, D.L. Penn

Treatment approaches with a special focus on social cognition: Overview and empirical results

V. Roder, A. Medalia (Eds.), Neurocognition and social cognition in schizophrenia patients, Karger, Basel, Switzerland (2010), pp. 61-78

Wykes et al., 2008

T. Wykes, C. Steele, B. Everitt, N. Tarrier

Cognitive behavior therapy for schizophrenia: Effect sizes, clinical models and methodological rigor

Schizophrenia Bulletin, 34 (2008), pp. 523-527

Höschel and Irle, 2001

K. Höschel, E. Irle

Emotional priming of facial affect identification in schizophrenia

Schizophrenia Bulletin, 27 (2) (2001), pp. 317-327 